\title{
Visibile narrare
}

\author{
Eduard Vilella
}

Universitat Autònoma de Barcelona

eduard.vilella@uab.cat

\begin{abstract}
L'Ekphrasis dantesca costituisce un capitolo fondamentale nella storia del fenomeno e in generale dei rapporti (in senso pratico e teorico) fra immagini e testo, d'altronde particolarmente importanti nel sistema culturale medievale. L'articolo si propone di esaminare brevemente la questione focalizzando l'attenzione nelle descrizioni delle immagini presenti in Purgatorio X. L'oggetto di interesse centrale è la loro implicita narratività, riguardo alla quale si cerca di stabilire dei possibili vincoli con le modalità narrative proprie delle arti visuali dell'epoca.
\end{abstract}

Parole chiave: Dante; Ekphrasis; immagine/testo; narrazione visiva.

Abstract. Visibile narrare.

Dante's is a prominent chapter both in the Ekphrasis story as such and more generally in the story of the (practical and theoretical) relationships between image and text (which were also particularly important in Medieval culture). This paper aims at briefly examining the question focusing on the image descriptions of Purgatorio X. The main subject of attention is the narrative modes which they implicitly carry and the possible links between these and the characteristic narrative modes of Medieval visual arts.

Keywords: Dante; Ekphrasis; image/text; visual narrative. 
Le celebri descrizioni delle immagini intagliate, all'altezza dei canti X-XII del Purgatorio, nella cornice dei superbi sulla parete e poi sulle lastre del pavimento, costituiscono, come ben si sa, un capitolo di straordinario interesse per quanto riguarda la storia dei rapporti fra immagine e testo, all'interno e anche oltre la tradizione classica dell' Ekphrasis in senso stretto. ${ }^{1}$ La perfezione divina di queste opere mette in risalto il contrasto tra la connotazione petrosa e rigida del peccato, dei peccatori, e del contrappasso ad essi corrispondente, e la duttilità sovrumana esibita dalle raffigurazioni nel loro presentarsi superiori a qualsiasi altra immaginabile e persino alla stessa Natura, di cui di solito l'arte dovrebbe essere imitatrice; nel loro manifestarsi, come nella famosa espressione, un visibile parlare che riesce a confondere le percezioni del personaggio che le contempla. ${ }^{2}$

Senz'altro si prospettano come il momento più intensamente e esplicitamente vincolante alle arti figurative della Commedia (e complessivamente dell'opera dantesca). Per un verso, anzi, a questo momento può essere attribuito un ruolo strategicamente centrale nel poema. Dato che si presentano come una sorta di paradigma assoluto di ciò che è un'opera d'arte, queste immagini diventano termine di raffronto tramite il quale è possibile una riflessione a più strati, che interessa complessivamente e in profondità tutti gli aspetti dell'attività artistica.

È logico che siano molteplici gli interventi critici di rilievo suscitati dalla questione. Diversi e fondamentali gli ambiti di interesse: dal rimando all'esperienza artistica dell'epoca nelle sue diverse sfumature; all'intreccio di parola e immagine nel medioevo di cui è aspetto fondamentale la tradizione dell'exemplum; alle problematiche vincolate alla mimesi e alla rappresentazione, in cui si vede coinvolto en abyme lo stesso testo dantesco; fino allo statuto stesso dell'opera d'arte in quanto orizzonte ultimo della riflessione... ${ }^{3}$ Per quanto riguarda

1. Gianni Venturi, «Una Lectura Dantis e l'uso dell'ecfrasi: Purgatorio X», in Gianni Venturi, Monica Farnetti (a cura di), Ecfrasi. Modelli ed esempi fra Medioevo e Rinascimento I, Roma: Bulzoni, 2004, p. 15-31, p. 15. Per uno sguardo complessivo sull'Ekphrasis nel medioevo, cfr. Haiko WANDHOFF, Ekphrasis. Kunstbeschreibungen und virtuelle Räume in der Literatur des Mittelalters, Berlin: De Gruyter, 2003.

2. Georges GüNTERT, «Canto X» in Michelangelo Picone (a cura di), Lectura Dantis Turicensis, Firenze: Franco Cesati, p. 139-155.

3. Molto abbondanti sono infatti i titoli di rilievo nella bibliografia sulla questione: Teodolinda Barolini, «Re-presenting What God Presented: The Arachnean Art of Dante's Terrace of Pride», Dante Studies, with the Annual Report of the Dante Society, 105, 1985, p. 43-62; Lucia BatTAGLia RicCi, "Come [...] le tombe terragne portan segnato': lettura del dodicesimo canto del Purgatorio", in Gianni Venturi, Monica Farnetti (a cura di), Ecfrasi. Modelli ed esempi fra Medioevo e Rinascimento I, Roma: Bulzoni, 2004, p. 33-63; Andreas Kablitz, "Jenseitige Kunst oder Gott als Bildhauer. Die Reliefs Dantes Purgatorio (Purg. X-XII)", in ID. (a cura di) Mimesis und Simulation, Freiburg: Rombach, 1998, p. 309-359; János Kelemen, «Ekphrasis, non-existing images, impossible figures: Purgatorio 10-12, Paradiso 33", in Antonio Sorella (a cura di), Boccaccio, Dante e Verdone, Firenze: Franco Cesati, 2016, p. 137-148; Maria Maslanka-Soro, "Non vide mei di me chi vide il vero": l'arte "verace" nel girone dei superbi come mise en abyme dell'arte dantesca" Atti del covegno della Nedanten Aprile 2017 (in stampa); James H. McGregor, «Reappraising Ekphrasis in 
strettamente l'Ekphrasis, Karlheinz Stierle considera l'esempio dantesco vera svolta sull'asse diacronico poiché, in confronto ai casi precedenti nella storia del fenomeno, vi ha parte essenziale il coinvolgimento del soggetto "spettatore" (per riprendere ancora la fondamentale idea di Piermario Vescovo). ${ }^{4}$ Senza abbandonare le salde coordinate che questi studi hanno tracciato, vorrei in queste pagine ritornare alla questione con delle brevi considerazioni sui potenziali risvolti dell'episodio purgatoriale per quel che concerne la generale capacità delle immagini di risolversi in racconto; e vorrei farlo non solo dalla prospettiva della narratività latamente implicita nei fenomeni di Ekphrasis ma specialmente in rapporto al contesto storico, nell'intento di riallacciare certi aspetti della sfera più immediata delle immagini dantesche alle modalità narrative ritrovabili nella iconografia medievale - partendo dall'idea ampiamente condivisa del vincolo fra esse e alcune opere artistiche dell'epoca. Fermo restando che si tratta di un tipo di legame osservabile solo in quanto tendenza, e non dal punto di vista della possibile ricerca di fonti. Su questo versante la ricerca ha stabilito solidamente quanto, da una parte, siano importanti i punti di contatto fra gli ambiti artistici interessati e quanto invece, dall'altra, sia inconcepibile cercar di materializzarle in un eventuale discorso di derivazioni concrete, di appunto "fonti" determinabili. Infatti, in modo generale, immagini e parole, immagini e racconti configurano una rete complessa e articolata di rapporti storicamente dinamici, nelle cui irradiazioni, talvolta folgoranti, si fa sentire il complesso e sfaccettato complesso di elementi di cui è composto un determinato sistema culturale. Questa circostanza è particolarmente rilevante per quel che concerne il Medioevo, nel cui sistema immagini e parole mantengono un rapporto di particolare contiguità. ${ }^{5}$ Lo studio della letteratura medievale costituisce su questo fronte un ambito di interesse che, a più riprese, si dimostra fondamentale; e la Commedia di Dante in questo senso ne offre uno degli esempi di spicco. ${ }^{6}$ L'episodio purgatoriale, per la sua calibrata esecuzione densa di rimandi, è in questo senso emblematico quanto

Purgatorio 10", Dante Studies, with the Annual Report of the Dante Society, n. 121, 2003, p. 25-41; Michelangelo PICONe, "Il cimento delle arti nella "Commedia". Dante nel girone dei superbi (Purgatorio X-XII)", in Dante e le arti visive, Milano: Unicopli, 2006, p. 81-107; Karlheinz Stierle, "Il sistema delle belle arti nel Purgatorio», in ID., Il grande mare del senso. Esplorazioni nella Commedia di Dante, Roma: Aracne, 2014; Pier Mario Vescovo, «Ecfrasi con spettatore (Dante, Purgatorio X-XVII)», Lettere Italiane, 45, 1993, p. 335-360.

4. Cfr. Stierle, op. cit., p. 239; Vescovo op. cit.

5. Cfr. Hans Belting, «Das Bild als Text. Wandmalerei und literatur im Zeitalter Dantes», in Hans Belting, Dieter Blume (a cura di), Malerei und Stadtkultur in der Dantezeit. Die Argumentation der Bilder, München: Hirmer, 1989, p. 23-64; Maria Bettetini, Figure di verità. La finzione nel Medioevo occidentale, Torino: Einaudi, 2004; Eugenio Burgio, Racconti di immagini. Trentotto capitoli sui poteri della rappresentazione nel Medioevo occidentale, Alessandria: Ed. dell'Orso, 2001; Rocío SÁnchez Ameijeiras, Los rostros de las palabras. Imágenes y teoría literaria en el Occidente medieval, Madrid: Akal, 2014; Meyer SHAPIRo, Palabras, escritos e imágenes. Semiótica del lenguaje visual, Madrid: Encuentro, 1998.

6. Lucia BATTAGLIA RICCI, "Visione e viaggio: tra immaginario visivo e invenzione letteraria» in Michelangelo Picone (a cura di), Dante da Firenze all'aldilà, Firenze: Cesati, 2001, p. 15-73. 
ai molteplici stimoli interpretativi che scaturiscono da una simile prospettiva. Nel caso di Dante poi, non va sottovalutato il rapporto privilegiato dell'autore, anche concreto, con l'ambito delle arti. Ma su questo aspetto dovremo tornare: comunque sia, è bene quindi ribadire l'osmosi che in partenza esiste fra i due ambiti in questione, quello delle immagini e quello dei testi, spesso considerati più distanti di quanto lo fossero in realtà; e quindi tenerne conto in quanto cornice ineludibile per la Commedia nella quale, infatti, come ha sottolineato Lucia Battaglia-Ricci, si osserva con singolare chiarezza quanto fosse permeata la cultura medievale da tradizioni di natura diversa da quella scritta. ${ }^{7} \mathrm{Nel}$ poema sacro insomma sono ben visibili le conseguenze di questo fitto tessuto di stimoli diversi, «inesausto gioco di interferenze, nel quale risulta impossibile decrittare crediti e debiti»; 8 e se Dante è immerso, infatti, in questa densa rete di relazioni, che lega tradizione figurativa e tradizione letteraria, non è detto invece che queste relazioni siano riportabili ad un eventuale discorso su fonti e influenze. Non è possibile dunque parlare «nei termini rigidi e rigorosi di un rapporto intertestuale tra "testi" precisi e individualizzabili» ma piuttosto di una relazione di tipo "interdiscorsivo", alla cui base si troverebbero difatti i diversi elementi di una rete di fenomeni culturali ampiamente condivisa. ${ }^{\text {? }}$

Fatte queste considerazioni, veniamo alle immagini purgatoriali. Si ricorderà come Dante personaggio contempli nel canto X gli intagli di meravigliosa fattura: ${ }^{10}$

Là sù non eran mossi i piè nostri anco, quand'io conobbi quella ripa intorno che dritto di salita aveva manco, esser di marmo candido e addorno d'intagli sì, che non pur Policleto, ma la natura lì avrebbe scorno.

Sono tre le scene di umiltà proposte in questi intagli. Nella prima, viene rappresentata l'Annunciazione, nella seconda, la danza di Davide davanti all'Arca, nella terza il noto episodio dell'imperatore romano Traiano e la povera vedova. A queste tre raffigurazioni di massima rilevanza, proposte come esempi di umiltà, si opporrà nel canto XII la consistente serie di esempi di superbia punita rappresentata sul pavimento della cornice, a modo delle lastre tombali. Qui ad ogni modo vorrei focalizzare l'attenzione nelle tre rappresentazioni del canto X.

Per tutti i tre casi, la perfezione artistica è assolutamente singolare, poiché in realtà gli intagli sono opera divina, e il risultato è tale da riuscire persino a con-

7. «Tradizione orale e tradizione visiva trasmettono infatti un "pacchetto" di nozioni e di competenze non meno importante e più diffusamente condiviso di quello trasmesso dalla tradizione scritta, con la quale esse stringono peraltro rapporti molto stretti», ibid., p. 16.

8. Ibid., p. 50 .

9. Ibid., p. 52

10. Si cita da Dante Alighieri, Commedia, (con il commento di Anna Maria Chiavacci LeONARdi), Bologna: Zanichelli, 1999. 
fondere la percezione di Dante personaggio. L'angelo della scena dell'Annunciazione "pareva si verace» (v. 37) «che non sembiava immagine che tace» (v. 39); similmente sembrano sentirsi le voci osannanti degli spettatori nella scena della danza di Davide (quindi facendo "discordi" i sensi di Dante personaggio):

Dinanzi parea gente; e tutta quanta, partita in sette cori, a' due mie' sensi faceva dir l'un 'No', l'altro 'Sì, canta'.

Similemente al fummo de li 'ncensi che v'era imaginato, li occhi e'l naso e al sì e al no discordi fensi.

Infine, nell'episodio di Traiano, la perfezione dell'intaglio fa sì che le bandiere si muovano al vento, che l'esercito circondante le due figure centrali sia "calcato e pieno", e che addirittura vengano riproposti come parte della raffigurazione i diversi momenti di un dialogo a più battute.

Come questo fenomeno, e per estensione quelli presentati per le altre due scene, sia possibile è spiegato nella terzina conclusiva dell'episodio, tramite il riferimento alla natura appunto divina delle opere, da cui scaturisce il famoso «visibile parlare» estraneo all'esperienza umana nell'aldiqua:

Colui che mai non vide cosa nova produsse esto visibile parlare, novello a noi perché qui non si trova.

È da qui che ha origine ed è stato possibile sviluppare da diversi studiosi, come si accennava prima, il paragone fra questa mimesi divina e la stessa arte letteraria di Dante che vi si rispecchia. Se si può infatti affermare che gli episodi dei canti X e XII del Purgatorio offrono degli esempi di Ekphrasis senz'altro fra i più notevoli della storia della letteratura medievale, ciò è così anche perché essi sembrano proporsi strategicamente come brani che vanno oltre l'immediato rimando all'ambito dell'ekphrasis e pure dell'exemplum, per emergere quali riflessioni di vasta portata per quanto riguarda lo statuto dell'arte umana e quindi anche le dinamiche profonde della rappresentazione e delle capacità intellettive: ${ }^{11}$ non a caso, afferma Vescovo, è plausibile osservare nella successione iniziata in questi canti e fino al XVII, una progressione che trova punto culminate nel riferimento alla fantasia non vincolata alla percezione e così alla prima apparizione del sintagma «alta fantasia». ${ }^{12}$

Ora, mi sembra che ci sia ancora spazio per riflettere su altri risvolti di questo singolarissimo potere di rappresentazione. Vorrei quindi soffermarmi sui tre esempi descritti ma dal punto di vista della narratività nel senso più diretto. Rivediamone allora lo sviluppo concreto. L'impostazione della scena dell'angelo è semplice e costruita su pochi elementi:

Langel che venne in terra col decreto de la molt'anni lagrimata pace,

11. Cfr. Barolini, op. cit; Maslanka-Soro, op. cit.; Picone, op. cit.

12. Vescovo, op. cit. 
ch'aperse il ciel del suo lungo divieto,

dinanzi a noi pareva sì verace quivi intagliato in un atto soave,

che non sembiava imagine che tace.

Giurato si saria ch'el dicesse 'Ave!';

perché iv'era imaginata quella

ch'ad aprir l'alto amor volse la chiave;

e avea in atto impressa esta favella

'Ecce ancilla Dei', propriamente

come figura in cera si suggella.

In quella di Davide davanti all'Arca, si può notare come, se fatto il paragone con il testo biblico, gli elementi narrativi interessati corrispondano a più momenti del racconto. La raffigurazione concentrerebbe narrativamente i due tempi del viaggio dell'Arca, nonché l'ulteriore rammarico di Micol —anche questo presentato originariamente in due tempi nel testo: ${ }^{13}$

Era intagliato lì nel marmo stesso

lo carro e' buoi, traendo l'arca santa,

per che si teme officio non commesso.

[...]

Lì precedeva al benedetto vaso,

trescando alzato, l'umile salmista,

e più e men che re era in quel caso.

Di contra, effigïata ad una vista

d'un gran palazzo, Micòl ammirava

sì come donna dispettosa e trista.

E si è già indicato come, infine, l'episodio della povera vedova e Traiano è risolto praticamente come la rappresentazione di un dialogo a più riprese $\mathrm{e}$ quindi, implicitamente, come rimando a una successione narrativa di una certa complessità, piuttosto che a un solo istante del racconto:

Quiv'era storiata l'alta gloria

del roman principato, il cui valore

mosse Gregorio a la sua gran vittoria;

i' dico di Traiano imperadore;

e una vedovella li era al freno,

di lagrime atteggiata e di dolore.

Intorno a lui parea calcato e pieno

13. Cfr. il commento di A.M. Chiavacci Leonard: "Dante racchiude in una sola "storia», come in un riquadro o formella scolpita, i due tempi successivi in cui si svolge il viaggio nel testo biblico e l'arrivo in Gerusalemme, secondo il testo biblico, "il re ballava davanti al Signore saltando con tutte le sue forze e aveva stretta alla cintura la veste sacerdotale" (II Sam. 6, 14)». E la scelta lessicale aggiunge la capacità per mimetizzare, nel caso di Micol, un ulteriore momento dello stesso racconto: «sembra probabile che le due parole di Dante vogliano tradurre le due indicazioni del versetto, anche perché la successiva figurazione di Micòl dispettosa e trista traduce nell'espressione del suo volto le parole da lei rivolte a David al suo rientro nel palazzo: "oh come fu glorioso oggi il re d'Israele, scoprendosi dinanzi alle serve dei servi e denudato come un buffone!"» 
di cavalieri, e l'aguglie ne l'oro sovr'essi in vista al vento si movieno.

La miserella intra tutti costoro pareva dir: «Segnor, fammi vendetta

di mio figliuol ch'è morto, ond'io m'accoro»;

ed elli a lei rispondere: «Or aspetta

tanto ch'i' torni»; e quella: «Segnor mio», come persona in cui dolor s'affretta, «se tu non torni?»; ed ei: "Chi fia dov'io, la ti farà»; ed ella: "L'altrui bene a te che fia, se 'l tuo metti in oblio?»; ond'elli: «Or ti conforta; ch'ei convene ch'i' solva il mio dovere anzi ch'i' mova: giustizia vuole e pietà mi ritene».

Questo mi sembra una fatto chiave. Da una parte, è evidente che, in virtù dell'economia inevitabilmente discorsiva del racconto dantesco, ma anche di quella natura sovrumana degli intagli, la complessità della narrazione degli episodi non presenta problemi particolari quanto alla plausibilità. Tuttavia, dall'altra, si potrebbe ugualmente ipotizzare che il "referente" (se mi è concessa l'espressione) fosse un racconto per immagini a sua volta complesso. A mio avviso insomma non è secondario notare che non si tratta di schemi narrativi alieni alla sensibilità medievale. Mi sembra interessante cioè insistere su come sia precisa la concordanza nei confronti del linguaggio delle immagini proprio dell'arte dell'epoca, la cui potenziale narratività poggia su fenomeni come la sintesi ma, anche, la simultaneità di elementi temporalmente eterogenei di una stessa diegesi. Dante sembra in realtà sviluppare i termini assoluti di una tendenza caratteristica nella cultura medievale.

In questo senso, come si accenava prima, è pertinente sottolineare la precisione dimostrata in genere da Dante per quel che riguarda le sue varie allusioni artistiche. Da questo punto di vista vanno ricordati i rimandi espliciti all'ambito delle arti visive, le quali possiedono un rilievo privilegiato nell'orizzonte di riferimento della Commedia. Certo, molto diretti sono gli esempi che ci occupano, o l'episodio da essi incorniciato, cioè l'incontro con Oderisi (Purgatorio $\mathrm{XI}$ ), in cui vengono sviluppate delle riflessioni che interessano gli artisti concreti (con richiamo a Giotto, Cimabue, Franco Bolognese), le loro produzioni, il loro essere soggetti alle dinamiche storiche. Ma non mancano esempi occasionali lungo tutta l'opera in cui si percepisce quanto fosse presente la cultura artistica in Dante. Pare ragionevole infatti che si possa sostenere una sua discreta competenza pratica sul fronte artistico, come testimoniato da diversi brani delle sue opere, sin dal celebre episodio che lo presenta intento a disegnare. $\grave{E}$ un aspetto che probabilmente sta a indicare che l'accuratezza del suo discorso risponde proprio a una vera competenza di tipo pratico e di un certo rilievo, ${ }^{14}$

14. Fortunato Bellonzi, «Arti figurative», in Enciclopedia Dantesca, Roma: Istituto della Enciclopedia Italiana p. 400-403; Umberto M. Milizia, «La concezione delle arti figurative in Dante», Folium n. 1/5, 1999, p. 8-20; MARIo PePE, «La letteratura artistica e le origini della 
per cui è evidente allora l'esattezza di riferimenti come, per esempio, quello di $\mathrm{Pg}$. XII

Qual di pennel fu maestro o di stile che ritraesse l'ombre e' tratti ch'ivi mirar farieno uno ingegno sottile? Morti li morti e i vivi parean vivi: non vide mei di me chi vide il vero, quant' io calcai, fin che chinato givi.

ma anche al diletto 'estetico' da parte del fruitore in $P d$. XXXI

E quasi peregrin che si ricrea nel tempio del suo voto riguardando, e spera già ridir com' ello stea, su per la viva luce passeggiando, menava io li occhi per li gradi, mo sù, mo giù e mo recirculando.

Se questo è il contesto storico e personale, diventa quindi interessante ribadire il collegamento, tutt'altro che generico, fra quello che si descrive nel testo e il suo ideale referente. Dico "ideale referente" nel senso che non intendo, va ribadito, aprire assolutamente un discorso sull'asse di eventuali "fonti" o "modelli" iconografici per il testo. Riprendo, per esplorare questo collegamento, le argomentazioni di James Mc Gregor, il quale sostiene che per i tre casi è necessario riferirsi a quelli che erano gli usi iconografici dell'epoca più che a una fonte scritta. Così, per quanto riguarda il primo episodio, gli elementi presenti nella descrizione dantesca potrebbero essere visti come ripresa dell'impostazione iconografica più abituale all'epoca. ${ }^{15}$ Nella scena dell'Annunciazione, infatti, mancano elementi narrativi altrove presenti quali la domestica e la colomba a simbolizzare il potere divino di concezione: orbene, la tradizione dei bassorilievi o sculture relative alla scena (ma non quella relativa ad altri tipi di figurazione quali affreschi e miniature) si caratterizzava proprio per questo particolare.

Nell'ambito delle sculture dunque «his [di Dante] reliance on gesture and little else to characterize the scene is true to the tradition». ${ }^{16} \mathrm{E}$ un'economia di risorse che segna chiare distanze nei confronti del racconto del Vangelo di Luca; il che, in breve, permette di affermare che le terzine mantengono una sintonia decisamente più palese, rispetto ai modelli iconografici usuali al tempo di Dante, di quanto non lo facciano con il testo che a un primo sguardo dovrebbe costituirne la "fonte" letteraria. Dice infatti McGregor quanto a questa forma condensata:

critica d'arte nell'età di Dante e nella cultura del Trecento", Cultura e scuola, n. 33/129, 1994, p. 74-85; Fernando Salsano, "Arte», in Enciclopedia Dantesca, Roma: Istituto della Enciclopedia Italiana, 1984, p. 397-399.

15. Op. cit.

16. Ibid., p. 29. 
[...] we willingly accept it as faithful to the text of Luke, though it is not. It is faithful instead to the iconographic tradition that compressed Luke's narrative into a simple, simultaneous coherent set of actions. ${ }^{17}$

Nel caso della seconda scena, il materiale è più complesso; tuttavia vi si può individuare una tendenza analoga. Qui sono diversi momenti del racconto che vengono evocati simultaneamente: cioè sviluppando gli spunti dell'eventuale immagine (superfluo insistere, parlo ipoteticamente) che potrebbe essere configurata da un insieme di elementi strategicamente condensati o presentati anche iterati in giustapposizione simultanea:

The Bible describes actions that unfolded over long spans of time and space, but Dante represented them by as if they occurred in a single place and a moment of time. In this presentation, he is again following a visual rather than a textual tradition. $[\ldots]^{18}$

In questo senso, la tradizione iconografica e il testo dantesco coinciderebbero nel presentare la narrazione biblica «as a set of simultaneous actions rather than a story that unfolds through time and space». ${ }^{19}$

Quanto alla terza scena, l'argomentazione di Mc Gregor cerca di riallacciarsi alle versioni della leggenda di San Gregorio, secondo le quali il santo fu commosso dalla personalità di Traiano dopo aver contemplato una raffigurazione in cui si faceva evidente la misericordia dell'imperatore. Dante in questo caso sembrerebbe poggiare su un racconto il cui argomento non parte direttamente dai fatti proposti nel testo, ma è focalizzato piuttosto sulla reazione di Gregorio. ${ }^{20}$ Una reazione che si fonda sulla capacità del santo "to intuit an extended narrative from a mute work of sculpture». L'opera di cui si parla potrebbe pure essere del tipo della colonna traiana o simili, nei cui programmi iconografici è lecito osservare potenziali candidati in proposito. ${ }^{21}$ Qui i legami con la tradizione dell'exemplum sarebbero molto evidenti. Comunque sia, ci troviamo davanti a (per così dire) una narrazione di una certa durata e complessità, risolta in una rappresentazione visuale di momenti presentati simultaneamente; in una narrazione che coinvolge diverse battute, che acquisiscono la dimensione temporale nella descrizione, che è verbale e, di necessità, temporale e sequenziale. ${ }^{22}$ Infatti, se la perfezione delle opere giustifica che si possa parlare di un "visibile parlare», che riesce a travalicare i limiti delle rappresentazioni visive materiali, va detto pure che in prima istanza non si può dimenticare quanto l'immagine statica sia suscettibile di articolarsi nel tempo della narrazione.

17. Ibid., p. 30 .

18. Ibid., p. 32.

19. Ibid., p. 32-33. E infatti, sostiene Mc. Gregor, questo rapporto di vicinanza è d'altronde argomentabile dai modi particolari tramite cui gli illustratori resero entrambe le scene, in certo senso più vincolabili alla tradizione visuale che al testo dantesco.

20. Cfr. GüNTERT, op. cit.

21. McGregor, op. cit., p. 34-38.

22. Cfr. Ernest B. Gilman, «Los estudios interartísticos y el "imperialismo" del lenguaje», in Antonio 
La costruzione delle battute deve essere intesa allora nella prospettiva di Dante personaggio che vede, e per così dire "legge" normalmente, le immagini ("normalmente" nel senso di ciò che era la consuetudine); personaggio in cui cioè si rispecchia l'esperienza più abituale del fruitore di immagini (e difatti ci si ritrova chiaramente di fronte a un caso di mise en abyme il cui oggetto è la setessa esperienza dell'arte, come indicava Battaglia Ricci).

Non va infatti dimenticato che, in modo chiaro nel caso dell'episodio di Davide, più tenuemente in quello del dialogo fra la vedova e Traiano, la tradizione iconografica poteva offrire numerosi esempi da cui partire, per immaginare un impianto artistico capace di una certa complessità temporale nella narrrazione. Possiamo quindi andare oltre alle ovvie condensazioni ed espansioni intrinseche alla dimensione verbale dei brani e ricordare anche quei numerosi casi in cui la capacità narrativa delle immagini si risolve in una diversità di episodi che, inevitabilmente, si presentano di forma simultanea alla percezione. ${ }^{23} \mathrm{Si}$ tratta di una modalità di narrazione molto frequente all'epoca, ma anche prima e dopo. Come ha stabilito Lew Andrews, contro quello che vorrebbe una impressione piuttosto diffusa ma non per quello sostenibile, la vitalità del fenomeno non corrisponde, come spesso si vorrebbe, a un modo di rappresentazione arcaico destinato a essere cancellato inevitabilmente con il perfezionamento delle tecniche pittoriche. La narrazione continua o simultanea non è, cioè, propria solo del Medioevo (e con esso, dell'antichità): è anzi particolarmente presente nel momento storico dello sviluppo della prospettiva in pittura, quando si cerca dunque di rappresentare la realtà in base al punto di vista di un osservatore, il quale ovviamente non potrebbe percepire simultaneamente due momenti narrativi successivi. Proprio il ' 400 in coincidenza con lo sviluppo della rappresentazione dello spazio costituirebbe uno dei momenti di ripresa più decisa di questa modalità narrativa. ${ }^{24}$

I secoli posteriori si sposteranno da questi usi e oggi, chi contempla le immagini è meno sensibile a questa potenziale dimensione dell'arte visiva, e soprattutto alla sua logica. ${ }^{25} \mathrm{Il}$ "visibile parlare», in conclusione, potrebbe rimandare anche, in forma di distillato essenziale, a fenomeni narrativi da rapportare decisamente all'esperienza artistica ed estetica dell'epoca di Dante: le modalità narrative, le elaborazioni verbali ad esse vincolabili, l'intimo legame che contraddistingue il rapporto stabilitosi fra le due sfere, i modi insomma di produzione e di ricezione delle immagini.

Monegal (a cura di), Literatura y pintura, Madrid: Arco Libros, 2000, p. 187-222, p. 197.

23. Lew Andrews, Story and space in Renaissance art: the rebirth of continuous narrative, Cambridge; New York: Cambridge University Press, 1995; François Garnier, Le Langage de l'image au moyen âge. II, Grammaire des gestes, Paris: Léopard d'or, 1989; Wolfgang KemP, "Narrativity» in Robert S. Nelson; Richard Shiff (a cura di) Critical Terms for Art History, Chicago: University of Chicago Press, 1996, p. 58-69; Marilyn A. Lavin, The place of narrative: Mural decoration in italian Churches, 431-1600. The work of art before the work of art, Chicago/London: The University of Chicago Press, 1990.

24. ANDREWs, op. cit.

25. Lavin, op. cit. 